\title{
Interactions Among Rumen Fermentation Characteristics, the Microbiota, and Host Gene Expression in Response to Ambient Temperature Fluctuations in Tibetan Sheep
}

\section{Weibing Lv}

Gansu Agricultural University https://orcid.org/0000-0002-2303-5180

Xiu Liu ( $\boldsymbol{D}$ liuxiu@gsau.edu.cn )

Gansu Agricultural University

\section{Yuzhu Sha}

Gansu Agricultural University

Hao Shi

Gansu Agricultural University

Hong Wei

Gansu Agricultural University

\section{Yuzhu Luo}

Gansu Agricultural University

\section{Jiqing Wang}

Gansu Agricultural University

\section{Shaobin Li}

Gansu Agricultural University

\section{Jiang Hu}

Gansu Agricultural University

\section{Xinyu Guo}

Gansu Agricultural University

\section{Xiaoning Pu}

Gansu Agricultural University

\section{Research}

Keywords: Rumen fermentation, Microbiota, 16S rRNA, Gene expression, Tibetan sheep

Posted Date: June 4th, 2021

DOI: https://doi.org/10.21203/rs.3.rs-572098/v1 
License: (c) (i) This work is licensed under a Creative Commons Attribution 4.0 International License. Read Full License 


\section{Abstract}

Background: As an important ruminant on the Qinghai-Tibet Plateau, Tibetan sheep can maintain their population reproduction rate in the harsh high-altitude environment of low temperature and low oxygen, which is related to their special adaptations to the plateau. Microbes (known as "second genomes") play an important role in the host adaptations. However, there have been no reports on the effects of the interactions among rumen fermentation, the microbiota, and host gene expression on the adaptation of Tibetan sheep to high altitude.

Results: Rumen fermentation characteristics, the microbiota, and rumen epithelial gene expression of Tibetan sheep in various months (February, April, June, August, October, and December) were analyzed. The results show that the rumen fermentation characteristics of Tibetan sheep differed in different months. The total SCFA, acetate, propionate, and butyrate concentrations were highest in Oct and lowest in Jun. The Cellulase (CL) activity was highest in Feb (when it was significantly higher than in Aug and Oct), while the acidic xylanase (ACX) activity was highest in Apr, followed by Jun (and it was significantly higher in both Apr and Jun than in Feb, Aug, and Oct). In addition, the diversity and abundance of rumen microbes differed in different months. Bacteroidetes (53.4\%) and Firmicutes (27.4\%) were the dominant phyla. Prevotella_ 1 and Rikenellaceae_RC9_gut_group were the dominant genera. The abundance of Prevotella_1 was highest in Jun (27.8\%) and lowest in Dec (17.8\%). Random forest analysis showed that Lachnospiraceae_NK3A20_group and Rikenellaceae_RC9_gut_group played important roles in rumen microbial composition in different months. In addition, the expression of CLAUDIN4 and ZO1 (ruminal epithelial barrier-related genes) was significantly higher in Apr than in Aug and Dec, while the expression of SGLT1 (nutrient absorption-related gene) was highest in Aug, but were significantly lower than CLAUDIN4 and ZO1 expression in the corresponding month. Correlation analysis showed that there were interactions among rumen fermentation characteristics.

Conclusions: There was a certain correlation between rumen fermentation products, the microbiota, and host gene expression. The microbiota, and host gene expression, and the host adjusted the rumen fermentation and microbiota structure according to changes in ambient temperature, to adapt to the plateau environment.

\section{Background}

The Qinghai-Tibet Plateau is the world's highest and largest plateau and the only plateau used for grazing in four seasons. It has a unique ecological environment, involving high altitude, low oxygen levels, strong ultraviolet rays, and severe imbalances in seasonal forage supply [1]. Livestock living on this plateau have special high-altitude adaptations that allow them to maintain their normal reproduction rate. The green-up stage (from early Apr to May), green grass stage (from Jun to Sep) and withered grass stage (from early Oct to early Mar, with rejuvenation occurring from Apr) of plateau grasslands reflect the changes in ambient temperature. The phenological changes in the plants of plateau grasslands significantly influence the grassland ecosystem, grazing livestock, and their gut microbes [2]. Under 
natural grazing conditions, the forage dry matter intake of Tibetan sheep during the green grass stage is $116 \mathrm{~g} / \mathrm{kgBW}^{0.75}$, and it drops to $59 \mathrm{~g} / \mathrm{kgBW}^{0.75}$ in the withered grass stage, while the. The corresponding crude protein (CP) level drops from $14.42-4.47 \%$ and the crude fiber (CF) level increases from $25.41-$ $32.57 \%$. Tibetan sheep mainly obtain nutrients by eating natural forages. During the withered grass stage, Tibetan sheep obtain nutrients only from withered grass, which allows them to maintain their basic metabolic and reproduction rates. They provide local herders with meat, wool, fuel (animal dung as fuel), and other daily necessities, making them indispensable economic animals for local herders.

The nutritional intake of ruminants on the plateau changes with dynamic changes in ambient temperature. Forage nutrition is an environmental factor that changes with the ambient temperature, and forage availability strongly influences the host's gut microbial composition [3,4]. The types and abundances of microbes in the host are associated with the host growth performance, physiological phenotype, and health status [5]. The gut microbiota regulates immune processes and metabolism using microbial metabolites (such as short-chain fatty acids [SCFAs]) to maintain homeostasis, regulate immune processes, and protect host health [6]. Changes in the gut microbiota composition or function may induce diseases in the host [7]. The rumen microbiota in ruminants is responsible for decomposing plant-derived forages to produce nutrients that can be directly used by the host. The microbes participate in host metabolism and exchange nutrients and information with the host. The microbiota composition is affected by various factors such as diet, species, host physiology, and the environment, among which diet is the most important factor [8]. The microbiota composition determines the specific fermentation pathways in the rumen [9]. The intestinal microbes have strong symbiotic relationships with macroorganisms [10], and they co-evolved with the host, playing important roles in forage fermentation and host energy supply [11]. The gut microbiota can help the host to decompose cellulose, hemicellulose, and other substances that the host cannot directly use, thereby providing the host with energy and nutrients [12].

The rumen epithelium is a unique place where the host interacts with its microbiota, and the microbiota affects the host's net nutrient utilization by providing nutrients [13]. Intestinal epithelial cells (IECs) are considered to be immune cells [14], and microbiota metabolites are closely related to these epithelial cells. For example, butyrate promotes intestinal homeostasis by regulating IECs [15]. The rumen epithelial tissue of ruminants has complex tight junctions (TJs) composed of intercellular protein complexes forming selective barriers that interact with the rumen microbiota [16]. CLAUDIN4 is a key TJ protein, playing a key role in the barrier function [17]. ZO1 was the first TJ protein to be discovered, and it colocalizes with CLAUDIN4 in the apical intercellular space between epithelial cells, playing a role in TJ formation and regulation [18].

Rumen microbes can metabolize complex carbohydrates into D-glucose, which is broken down into SCFAs. High-affinity SGLT1 in the apical side of the sheep rumen epithelium can actively transport Dglucose from the rumen to the blood, so that the rumen epithelium maintains the ability to absorb glucose [19]. There is a close relationship between the rumen microbiota and the expression of host 
nutrient absorption- and barrier-related genes, and the rumen microbiota influences the host's nutrient absorption, energy utilization, and ruminal homeostasis.

However, there are very few studies on the impact of dynamic changes in ambient temperature on the rumen microbiota and rumen fermentation function of Tibetan sheep, or their interactions with the expression of rumen epithelial nutrient absorption- and epithelial barrier-related genes. Therefore, this study aimed to explore the changes in the rumen fermentation function and microbiota structure of grazing Tibetan sheep across a year on the Qinghai-Tibet Plateau, as well as the expression of nutrient absorption- and barrier-related genes in the rumen epithelium. We comprehensively analyzed the effects of the interactions among the rumen fermentation, the microbiota, and host gene expression on the adaptation of Tibetan sheep to the plateau, providing a reference regarding the adaptation mechanisms of Tibetan sheep on the plateau.

\section{Results}

\section{Rumen fermentation parameters}

The rumen fermentation parameters in the Tibetan sheep are shown in Table 1. The SCFA concentrations in the rumen of Tibetan sheep differed in different months. The total SCFA concentration was significantly higher in Oct than in other months $(P<0.05)$. The acetate, propionate, and butyrate concentrations peaked in different months, and the sum of the three accounted for $>83.1 \%$ of the total SCFAs. The acetate concentration was highest in Oct and lowest in Jun, and significantly higher in Oct and Dec than in other months $(P<0.05)$ and significantly lower in Jun than in other months $(P<0.05)$. The propionate concentration was significantly higher in Oct than in other months $(P<0.05)$, and lowest in Jun. The butyrate concentration was significantly higher in Aug and Oct than in other months $(\mathrm{P}<$ $0.05)$, and significantly lower in Jun than in other months $(P<0.05)$. The isobutyrate, isovalerate, and valerate concentrations were significantly higher in Feb and Apr than in other months $(P<0.05)$, and lowest in Dec. The acetate:propionate (A:P) was significantly higher in Dec than in other months $(\mathrm{P}<$ 0.05), and lowest in Feb. In addition, the $\mathrm{NH}_{3}-\mathrm{N}$ level in the rumen fluid was significantly higher in Jun and Aug than in other months, and was lowest in Feb. The CL and ACX activities in the rumen fluid also differed in different months. More specifically, CL activity was highest in Feb (when it was significantly higher than in Aug and Oct), and ACX activity was highest in Apr (and it was significantly higher in both Apr and Jun than in other months) and lowest in Aug. 
Table 1

Results of determination of rumen fermentation parameters in different periods

\begin{tabular}{|c|c|c|c|c|c|c|}
\hline $\begin{array}{l}\text { Fermentation } \\
\text { parameters }\end{array}$ & Feb & Apr & Jun & Aug & Oct & Dec \\
\hline Acetate $(\mathrm{mmol} / \mathrm{L})$ & $\begin{array}{l}23.56 \pm \\
0.33^{d}\end{array}$ & $\begin{array}{l}26.08 \pm \\
0.81^{\mathrm{C}}\end{array}$ & $\begin{array}{l}19.53 \pm \\
1.94^{\mathrm{e}}\end{array}$ & $\begin{array}{l}36.63 \pm \\
0.33^{b}\end{array}$ & $\begin{array}{l}40.36 \pm \\
0.88^{a}\end{array}$ & $\begin{array}{l}40.01 \pm \\
2.01^{\mathrm{a}}\end{array}$ \\
\hline Propionate(mmol/L) & $\begin{array}{l}12.72 \pm \\
0.61^{c}\end{array}$ & $\begin{array}{l}13.55 \pm \\
1.02^{\mathrm{bc}}\end{array}$ & $\begin{array}{l}10.33 \pm \\
0.30^{d}\end{array}$ & $\begin{array}{l}14.37 \pm \\
0.37^{b}\end{array}$ & $\begin{array}{l}15.96 \pm \\
0.49^{a}\end{array}$ & $\begin{array}{l}13.86 \pm \\
0.31^{b c}\end{array}$ \\
\hline Butyrate(mmol/L) & $\begin{array}{l}6.85 \pm \\
0.39^{c}\end{array}$ & $\begin{array}{l}7.48 \pm \\
0.45^{\mathrm{b}}\end{array}$ & $\begin{array}{l}5.71 \pm \\
0.20^{d}\end{array}$ & $\begin{array}{l}8.11 \pm \\
0.10^{\mathrm{a}}\end{array}$ & $\begin{array}{l}8.14 \pm \\
0.49^{a}\end{array}$ & $\begin{array}{l}6.05 \pm \\
0.19^{d}\end{array}$ \\
\hline Isobutyrate(mmol/L) & $\begin{array}{l}1.18 \pm \\
0.11^{\mathrm{a}}\end{array}$ & $\begin{array}{l}1.27 \pm \\
0.09^{a}\end{array}$ & $\begin{array}{l}0.97 \pm \\
0.02^{b}\end{array}$ & $\begin{array}{l}1.00 \pm \\
0.01^{\mathrm{b}}\end{array}$ & $\begin{array}{l}0.93 \pm \\
0.05^{\mathrm{bc}}\end{array}$ & $\begin{array}{l}0.82 \pm \\
0.01^{c}\end{array}$ \\
\hline Isovalerate(mmol/L) & $\begin{array}{l}2.53 \pm \\
0.11^{b}\end{array}$ & $\begin{array}{l}2.77 \pm \\
0.26^{a}\end{array}$ & $\begin{array}{l}1.85 \pm \\
0.03^{c}\end{array}$ & $\begin{array}{l}1.87 \pm \\
0.01^{c}\end{array}$ & $\begin{array}{l}1.69 \pm \\
0.12^{c}\end{array}$ & $\begin{array}{l}1.44 \pm \\
0.01^{d}\end{array}$ \\
\hline Valerate(mmol/L) & $\begin{array}{l}5.05 \pm \\
0.35^{a}\end{array}$ & $\begin{array}{l}4.61 \pm \\
0.21^{\mathrm{b}}\end{array}$ & $\begin{array}{l}4.26 \pm \\
0.12^{c}\end{array}$ & $\begin{array}{l}4.13 \pm \\
0.13^{\text {cd }}\end{array}$ & $\begin{array}{l}3.8 \pm \\
0.06^{d}\end{array}$ & $\begin{array}{l}3.08 \pm \\
0.15^{\mathrm{e}}\end{array}$ \\
\hline$A: P$ & $\begin{array}{l}1.85 \pm \\
0.06^{c}\end{array}$ & $\begin{array}{l}1.93 \pm \\
0.08^{\mathrm{c}}\end{array}$ & $\begin{array}{l}1.89 \pm \\
0.13^{c}\end{array}$ & $\begin{array}{l}2.55 \pm \\
0.04^{b}\end{array}$ & $\begin{array}{l}2.53 \pm \\
0.02^{b}\end{array}$ & $\begin{array}{l}2.88 \pm \\
0.08^{a}\end{array}$ \\
\hline Total SCFAs(mmol/L) & $\begin{array}{l}51.88 \pm \\
1.91^{\mathrm{d}}\end{array}$ & $\begin{array}{l}55.77 \pm \\
2.43^{\mathrm{c}}\end{array}$ & $\begin{array}{l}42.65 \pm \\
2.62^{\mathrm{e}}\end{array}$ & $\begin{array}{l}66.11 \pm \\
0.94^{b}\end{array}$ & $\begin{array}{l}70.91 \pm \\
1.64^{\mathrm{a}}\end{array}$ & $\begin{array}{l}65.27 \pm \\
2.37^{b}\end{array}$ \\
\hline $\mathrm{NH}_{3}-\mathrm{N}(\mathrm{mg} / \mathrm{dL})$ & $\begin{array}{l}2.38 \pm \\
0.03^{\mathrm{e}}\end{array}$ & $\begin{array}{l}5.82 \pm \\
0.08^{\mathrm{c}}\end{array}$ & $\begin{array}{l}9.54 \pm \\
0.26^{\mathrm{a}}\end{array}$ & $\begin{array}{l}8.10 \pm \\
0.02^{b}\end{array}$ & $\begin{array}{l}5.98 \pm \\
0.03^{c}\end{array}$ & $\begin{array}{l}3.37 \pm \\
0.03^{d}\end{array}$ \\
\hline $\mathrm{CL}$ activities $(\mu \mathrm{g} / \mathrm{min} / \mathrm{ml})$ & $\begin{array}{l}122.78 \pm \\
17.25^{a}\end{array}$ & $\begin{array}{l}110.82 \pm \\
15.58^{\mathrm{ab}}\end{array}$ & $\begin{array}{l}110.13 \\
\pm 6.5^{\mathrm{ab}}\end{array}$ & $\begin{array}{l}96.23 \pm \\
2.01^{b}\end{array}$ & $\begin{array}{l}99.21 \pm \\
4.85^{b}\end{array}$ & $\begin{array}{l}109.52 \pm \\
5.58^{\mathrm{ab}}\end{array}$ \\
\hline $\begin{array}{l}\text { ACX } \\
\text { activities(nmol/min/mL) }\end{array}$ & $\begin{array}{l}144.61 \pm \\
27.04^{c}\end{array}$ & $\begin{array}{l}293.32 \pm \\
20.78^{a}\end{array}$ & $\begin{array}{l}257.4 \pm \\
23.88^{\mathrm{a}}\end{array}$ & $\begin{array}{l}107.64 \pm \\
18.64^{d}\end{array}$ & $\begin{array}{l}162.19 \pm \\
14.37^{\mathrm{bc}}\end{array}$ & $\begin{array}{l}194.42 \pm \\
13.55^{\mathrm{b}}\end{array}$ \\
\hline
\end{tabular}

\section{Characteristics of rumen microflora in different months}

Rumen microbial 16S rRNA sequencing results and alpha and beta diversity

16S rRNA sequencing was performed on 36 samples obtained in different months, and 2,879,764 pairs of reads were obtained. After paired-end read merging and filtering, 2,773,869 clean tags were produced. Each sample produced at least 73,683 clean tags, with a mean of 77,052 , and a mean sequence length of 419 bp. Using Usearch software to the cluster tags (based on a 97\% similarity level), 1,223 OTUs were 
obtained, comprising 1,150, 1,168, 1,064, 1,149, 1,155, and 1,168 in Feb, Apr, Jun, Aug, Oct, and Dec, respectively. There were 941 OTUs that were shared by samples from Tibetan sheep rumens obtained in different months, and one unique OTU was found in the Dec samples, as shown in the Venn diagram in Fig. 1A. The rarefaction curve (Fig. 1B) plateaued at 20,000 reads, that is, the number of species did not significantly increase with increases in the number of tags sampled, indicating saturation. The sequencing coverage rate reached $>99.6 \%$, with the sequencing data authentically and reliably reflecting the samples (Table 2).

The number of OTUs in each month was highest in Oct and lowest in Jun, and significantly higher in Aug and Oct than in other months $(P<0.05)$, and significantly lower in Jun than in other months $(P<0.05)$. The Shannon index was significantly higher in Aug, Oct, and Apr than in other months $(P<0.05)$. The Simpson index exhibited no significant differences in different months. The abundance-based coverage estimator (ACE) and Chao1 index (representing the abundances of rumen microbes) was significantly higher in Aug and Oct than in other months $(P<0.05)$, and lowest in Jun. Principal coordinates analysis (PCOA) indicated obvious clustering related to the month of sample collection, with the Aug and Oct samples having similar rumen microbial diversity, the Feb and Apr samples having similar rumen microbial diversity, and the Jun samples having quite different rumen microbial diversity compared to the other months (Fig. 1C). Analysis of similarities (ANOSIM) indicated that the difference regarding samples from different months was significantly greater than the difference regarding samples from the same month, the reliability of the sequencing data was high, and subsequent analysis could be performed (Fig. 1D).

Table 2

alpha index diversity

\begin{tabular}{|c|c|c|c|c|c|c|}
\hline $\begin{array}{l}\text { Alpha } \\
\text { index }\end{array}$ & Shannon & Simpson & ACE & Chao1 & OTU & Coverage(\%) \\
\hline Feb & $\begin{array}{l}5.2411 \pm \\
0.1151^{c}\end{array}$ & $\begin{array}{l}0.0202 \pm \\
0.0043\end{array}$ & $\begin{array}{l}940.54 \pm \\
13.3818^{c}\end{array}$ & $\begin{array}{l}953.76 \pm \\
14.1885^{d}\end{array}$ & $882^{c}$ & 99.6 \\
\hline Apr & $\begin{array}{l}5.5801 \pm \\
0.052^{\mathrm{ab}}\end{array}$ & $\begin{array}{l}0.0104 \pm \\
0.0007\end{array}$ & $\begin{array}{l}1000.69 \pm \\
24.7213^{b}\end{array}$ & $\begin{array}{l}1015.56 \pm \\
24.3951^{\mathrm{c}}\end{array}$ & $952^{b}$ & 99.6 \\
\hline Jun & $\begin{array}{l}5.241 \pm \\
0.1235^{c}\end{array}$ & $\begin{array}{l}0.0194 \pm \\
0.0067\end{array}$ & $\begin{array}{l}891.69 \pm \\
17.8006^{d}\end{array}$ & $\begin{array}{l}909.56 \pm \\
18.8136^{d}\end{array}$ & $819^{d}$ & 99.6 \\
\hline Aug & $\begin{array}{l}5.6735 \pm \\
0.0755^{a}\end{array}$ & $\begin{array}{l}0.0113 \pm \\
0.0014\end{array}$ & $\begin{array}{l}1067.62 \pm \\
16.5539^{a}\end{array}$ & $\begin{array}{l}1076.28 \pm \\
15.8911^{\mathrm{ab}}\end{array}$ & $1027^{a}$ & 99.7 \\
\hline Oct & $\begin{array}{l}5.6725 \pm \\
0.0705^{a}\end{array}$ & $\begin{array}{l}0.0113 \pm \\
0.0018\end{array}$ & $\begin{array}{l}1073.34 \pm \\
14.2175^{\mathrm{a}}\end{array}$ & $\begin{array}{l}1092.61 \pm \\
13.2138^{a}\end{array}$ & $1030^{\mathrm{a}}$ & 99.7 \\
\hline Dec & $\begin{array}{l}5.3535 \pm \\
0.0256 b^{c}\end{array}$ & $\begin{array}{l}0.0179 \pm \\
0.0023\end{array}$ & $\begin{array}{l}1010.09 \pm \\
11.0127^{\mathrm{b}}\end{array}$ & $\begin{array}{l}1031.25 \pm \\
12.086 b^{c}\end{array}$ & $948^{b}$ & 99.6 \\
\hline
\end{tabular}


There were 17 phyla, 26 classes, 38 orders, 59 families, 149 genera, and 163 species. Bacteroidetes (> $53.4 \%$ ) and Firmicutes ( $>27.4 \%$ ) were the dominant bacterial phyla, accounting for $>83.3 \%$ of the total abundance in each month (Fig. $2 A$ ). The Firmicutes:Bacteroidetes $(F: B)$ ratio was significantly higher in Aug (0.619) than in Apr (0.500) and Dec (0.483). The abundance of Patescibacteria was $>1 \%$ in each month, and there were significant differences in different months $(P<0.05)$, with the highest abundance in Feb (8.17\%) and the lowest in Jun (1.09\%). Furthermore, as shown in the rank sum test chart, there were 12 different species at the phylum level (Fig. 2B). In particular, Firmicutes was significantly higher in Jun than in Feb and Dec $(P<0.05)$. Patescibacteria was significantly lower in Jun than in Feb and Dec $(P$ $<0.05$ ), while Kiritimatiellaeota exhibited the opposite trend. Elusimicrobia and Cyanobacteria were both significantly lower in Jun than in Feb and Apr $(P<0.05)$. Bacteroidetes had the highest abundance but exhibited no significant differences in different months.

There were 108 different species in 149 genera $(P<0.05)$. The abundances of Prevotella_ 1 , Rikenellaceae_RC9_gut_group, Christensenellaceae_R-7_group, Ruminococcaceae_NK4A214_group, and Prevotellaceae_UCG-001 were $>1 \%$ in each month (Fig. 2C). The dominant bacteria genera in each month were Prevotella_1 (>17.8\%; highest in Jun [27.8\%] and lowest in Dec [17.8\%]) and Rikenellaceae_RC9_gut_group (> 7.2\%; highest in Dec [25.8\%] and lowest in Aug [7.2\%]).

There were 12, 5, and 12 significantly differential species between Apr and Aug (rejuvenating grass vs green grass), Apr and Dec (rejuvenating grass vs withered grass), and Aug and Dec (green grass vs withered grass), respectively were found. According to the rank sum test chart (showing the top 20 species with the lowest P values at the genus level), Ruminiclostridium_9, Family_XIIIUCG-001, and Acinetobacter were significantly different between Apr and Aug; there were no significantly different genera between Apr and Dec; and Rikenellaceae_RC9_gut_group, DNF00809, Coprococcus_1, and Acinetobacterwere significantly different between Aug and Dec (Fig. 2D). In addition, Butyrivibrio_2, Moryella and Shuttleworthia were significantly higher in Jun than in Feb and Dec $(P<0.05)$; Olsenella was significantly lower in Feb than in Aug and Oct; Anaeroplasma and Sphaerochaeta were significantly lower in Jun than in Feb and Apr; and Acetitomaculum was significantly higher in Oct than in Feb and Dec. Random forest analysis at the genus level showed that Lachnospiraceae_NK3A20_group was the most important for sample classification, followed by DNF00809, Rikenellaceae_RC9_gut_group, and FD2005 (Fig. 3).

Microbe biomarkers in different months

To identify biomarkers that could significantly differentiate between different months, Linear discriminant analysis (LDA) effect size (LEfSe) analysis was performed (Fig. 4). Based on Linear discriminant analysis (LDA) scores > 4, the identified biomarkers for Feb, Apr, Jun, Aug, Oct, and Dec were Patescibacteria; uncultured_bacterium_f_F082; Firmicutes, Synergistetes, Prevotella_1, Butyrivibrio_2, Succiniclasticum, Ruminococcaceae_NK4A214_group, and Fretibacterium; Prevotellaceae_UCG-003; Prevotellaceae_UCG- 


\section{Prediction of rumen microbial gene function}

Next, PICRUSt software was used for gene function prediction based on the ruminal microbe 16S rRNA sequencing data.

Among the 46 Kyoto Encyclopedia of Genes and Genomes (KEGG) gene families and 25 Clusters of Orthologous Groups of proteins (COG) functional genes identified in the Apr and Aug samples (rejuvenating and green grass stages, respectively), METABOLISM pathway-related genes accounted for $>80.2 \%$ and $>37.12 \%$, respectively. The differential KEGG pathway analysis showed that there were 17 differential functional genes, among which METABOLISM pathway-related genes accounted for $67.56 \%$. In particular, Carbohydrate metabolism was significantly higher in Aug than in $\operatorname{Apr}(P=0.028)$, while Amino acid metabolism pathway $(P=0.019)$ and Energy metabolism pathway $(P=0.003)$ were significantly lower. The differential COG function prediction analysis showed that there were 9 differential functional genes, among which METABOLISM pathway-related genes accounted for $13.67 \%$. In particular, Coenzyme transport and metabolism was significantly higher in Apr than in Aug $(P=0.03)$.

Among the 43 KEGG gene families and 24 COG functional genes identified in the Apr and Dec samples (rejuvenating and withered grass stages, respectively), METABOLISM pathway-related genes accounted for $>80.6 \%$ and $>37.16 \%$, respectively. The differential KEGG pathway analysis showed that there were no significant differences in METABOLISM pathways. The differential COG function prediction analysis showed that there were 3 differential functional genes. In particular, the Amino acid transport and metabolism pathway (7.63\%) was significantly higher in Apr than in $\operatorname{Dec}(P=0.018)$.

Among the 43 KEGG gene families and 24 COG functional genes identified in the Aug and Dec samples (green and withered grass stages, respectively), METABOLISM pathway-related genes accounted for > $80.2 \%$ and $>37.12 \%$, respectively. The differential KEGG pathway analysis showed that there were 24 differential functional genes, among which METABOLISM pathway-related genes accounted for $>57.4 \%$. In particular, the Amino acid metabolism pathway (6.89\%) was significantly higher in Dec than in Aug (P $=0.019)$. The differential COG function prediction analysis showed that there were 9 differential functional genes, among which METABOLISM pathway-related genes accounted for $>15.90 \%$. In particular, the Amino acid transport and metabolism pathway $(7.63 \%)$ was significantly higher in Aug than in $\operatorname{Dec}(P=0.003)$.

\section{Expression of rumen epithelial nutrient absorption- and barrier-related genes in different months}

The expression levels of nutrient absorption and barrier-related genes in the rumen epithelium in different months are shown in Fig. 5. CLAUDIN4 expression was higher than the expression of the other assessed 
genes in each month assessed, and the trends in CLAUDIN4 and ZO1 were the same. CLAUDIN4 expression was significantly higher in Apr than in Aug and Dec (3.04- and 30.9-fold, respectively). ZO1 expression was 2.11- and 4.19-fold higher in Apr than in Aug and Dec, respectively. SGLT1 expression was highest in Aug, which was 3.37- and 3.50-fold higher than that in Apr and Dec, respectively.

\section{Interactions among rumen fermentation, the microbiota, and host gene expression in Tibetan sheep}

A correlation heatmap (correlation threshold $>0.5$ ) of the top 20 species (with high abundances of their corresponding genera in the rumen of Tibetan sheep) and fermentation parameters (SCFA and $\mathrm{NH}_{3}-\mathrm{N}$ levels and $C L$ and $A C X$ activities) and gene expression $\left(2^{-\Delta \Delta C T}\right)$ is shown in Fig. 6 . There were significant correlations between rumen fermentation parameters and genera $(P<0.05)$, with 33 positive and 20 negative correlations. Among these correlations, acetate was significantly positively correlated with Fretibacterium, Ruminococcaceae_NK4A214_group, and Rikenellaceae_RC9_gut_group. Propionate was significantly positively correlated with Prevotellaceae_UCG-003, and significantly negatively correlated with Ruminococcus_1. Butyrate was significantly positively correlated with Prevotella_ 1 and Succiniclasticum, and significantly negatively correlated with Rikenellaceae_RC9_gut_group and Ruminococcus_ 1 . $\mathrm{NH}_{3}-\mathrm{N}$ level was significantly positively correlated with Ruminococcaceae_NK4A214_group and Succiniclasticum, and significantly negatively correlated with Ruminococcus_ 1 and Rikenellaceae_RC9_gut_group. ACX activity was significantly positively correlated with Ruminococcus_1, Rikenellaceae_RC9_gut_group, and [Eubacterium]_coprostanoligenes_group, and significantly negatively correlated with Prevotellaceae_UCG-003.

The expression levels of nutrient absorption- and barrier-related genes were significantly correlated with bacterial genera $(P<0.05)$, with 12 positive and 8 negative correlations. Among these correlations, Ruminococcaceae_NK4A214_group and Succiniclasticum were significantly positively correlated with SGLT1 expression; Rikenellaceae_RC9_gut_group and Ruminococcus_ 1 were significantly negatively correlated with $S G L T 1$ expression $(P<0.01)$; Succiniclasticum was significantly positively correlated with CLAUDIN4 and ZO1 expression; and Fretibacterium and Rikenellaceae_RC9_gut_group were significantly negatively correlated with CLAUDIN4 and ZO1 expression.

\section{Discussion}

As an important ruminant in the Qinghai-Tibet Plateau, Tibetan sheep graze naturally throughout the year, and mainly obtain nutrients by eating natural forages. In ruminants, $>75 \%$ of SCFAs produced by rumen fermentation are absorbed via the rumen epithelium as the main host energy source [20]. We found that the total SCFA, propionate and butyrate were higher in Aug and Oct, and the propionate and butyrate were significantly higher than in other months; $\mathrm{NH}_{3}-\mathrm{N}$ levels were significantly higher in Jun and Augr than in 
other months. Forages in the green grass stage have high protein and carbohydrate content [21], and Tibetan sheep can produce high $\mathrm{NH}_{3}-\mathrm{N}$ levels after eating them by increasing the level of the main rumen community in the rumen [22]. In this study, in Aug, the Qinghai-Tibet Plateau was in the green grass stage, with rich pasture species and high nutrient levels. Therefore, In June, $\mathrm{NH}_{3}-\mathrm{N}$ mainly providing energy for the host, and in August and October, the total SCFA concentrations providing energy for the host. Regarding energy utilization, the A:P ratio is inversely proportional to the energy utilization efficiency of forage [23], indicating that the energy utilization efficiency of Tibetan sheep is lowest in Dec and highest in Apr. During the green grass stage, Tibetan sheep rapidly eat forages with sufficient nutrient levels within a small range [24], so metabolism decreases and more energy is stored by the body. In contrast, in the withered grass stage, Tibetan sheep need to travel across a larger area to search for forages, which increases metabolism and thereby decreases energy storage. In addition, ACX and CL activities in the rumen gradually increased from Dec, peaking in Jun and Apr, respectively. In the withered grass stage in plateau grasslands (from early October to early Apr), the cellulose proportion in the forages is higher than that of green grass. The increased ACX and CL activities allow more high-cellulose-containing substrates to be degraded into glucose that can be directly used by the host, in order to provide energy for the host to adapt to the reduced energy supply during this stage.

The rumen microbes of Tibetan sheep and their hosts have evolved a relatively stable microbiota structure during long-term co-evolution in order to adapt to the harsh environment of the Qinghai-Tibet Plateau. Among the many factors affecting the rumen microbiota structure, diet is key [25]. In this study, there were differences in the microbial abundances and diversity in the rumen of Tibetan sheep in different months. In particular, the microbial diversity was significantly lower in Dec than in other months, and the microbial abundance was significantly higher in Aug than in other months. The analyses of KEGG gene families and COG functional genes of microorganisms found that $>57.4 \%$ of the genes that were differentially expressed among different months were related to the METABOLISM pathway. Among them, the Carbohydrate metabolism pathway had the most significant impact regarding the differences between months, followed by the Amino acid and Energy metabolism pathways. This reflects the fact that rumen microbes and forage supply affect the METABOLISM pathway (mainly the Carbohydrate, Amino acid, and Energy metabolism pathways), which can affect host gene expression.

At the phylum level, both Bacteroidetes and Firmicutes were dominant (> 83.3\%) in each month, and the abundance of Bacteroidetes was higher than that of Firmicutes. This is consistent with the results reported by Liu et al [22]. However, Kim et al. [26] found that the mean abundances of Bacteroides and Firmicutes in the rumens of low-altitude livestock were only $31 \%$ and $56 \%$, respectively. Cunha et al. [27] also found that the mean abundances of Bacteroides and Firmicutes in goats in semi-arid regions of Brazil were only about $37.9 \%$ and $56.3 \%$, respectively. In the current study, there were no significant differences in the abundance of Bacteroidetes in different months, and it only increased in Dec. Members of this phylum can effectively decompose protein and carbohydrates in feed into SCFAs, providing energy for the host [28], and they also promote rumen growth and increase its volume [8]. The abundance of Firmicutes was significantly higher in Jun than in Feb and Dec. Firmicutes can help cells absorb glucose 
[8]. The increase in the abundance of Bacteroidetes in Dec indicates that it plays a more important role than Firmicutes in the high-altitude adaptation of the host during the period of nutrient deficiency. The abundances of Bacteroidetes and Firmicutes in the rumen of Tibetan sheep were maintained at stable levels during certain periods (Aug to Oct) of the year, which is of great significance to the stability of the rumen internal environment. The increase in the abundance of Bacteroidetes in Dec may have promoted rumen development and forage decomposition, thereby providing a certain amount of energy for the host. The significant increase in the abundance of Firmicutes in Jun may have helped the host to obtain more energy.

At the genus level, Prevotella_1 and Rikenellaceae_RC9_gut_group were dominant. Prevotella_1 plays an important role in the degradation and utilization of plant non-cellulosic polysaccharides, including starch, xylan, and protein [23]. Reduced protein and starch levels decrease the abundance of Prevotella_1 [29]. In this study, the abundance of Prevotella_ 1 was highest in Jun and lowest in Dec, which may be due to the lower protein content of Dec hay stage forages. Rikenellaceae_RC9 is closely related to members of the Alipites family [30], and it may play a role in the degradation of plant-derived polysaccharides [31]. In this study, Rikenellaceae_RC9_gut_group had the highest abundance in Dec, which may have increased highcellulose forage degradation, thereby providing energy for the host.

In addition, many cellulolytic bacterial genera [32] were found in this study, such as Ruminococcus_2, Fibrobacter, Butyrivibrio_2, Treponema_2, and Pseudobutyrivibrio. Cellulolytic bacteria can degrade cellulose in the rumen and play key roles in SCFA production [33]. Microorganisms have a rich library of protein-coding genes that can encode various enzymes related to metabolism [34]. Changes in the abundances of microbes lead to changes in enzymes, which alter the fermentation in the rumen [35]. ACX and $C L$ activities dropped to its lowest level in Aug, ACX activity was significantly lower in Aug than in other months, while CL activity remained at a stable level (from Apr to Dec), which indicates that the digestion and utilization of cellulose in forage grass by ruminal microorganisms remained relatively stable. It also implies that after a long period of co-evolution, a relatively stable cellulolytic community exists in the rumen of Tibetan sheep. We speculate that the microorganisms encoding cellulase proteins are an important part of the "core microbiota" [36] in the rumen of Tibetan sheep, and cellulolytic bacteria play an important role in the plateau adaptation mechanism of Tibetan sheep.

Studies have shown that high abundances of Prevotella and low abundances of Methanobacter in the rumen of Tibetan sheep promote forage fermentation to produce high SCFA concentrations and reduce methane production to avoid energy loss [37]. In this study, the total SCFA, propionate, and butyrate were higher in Aug to Oct, and SGLT1 expression in the rumen epithelium was also significantly increased. Butyrate is the main source of ruminant metabolic energy [38,39], and both propionate and butyrate can promote the development of rumen papilla [8]. Prevotella_1 was significantly positively correlated with butyrate level $(\mathrm{P}<0.05)$; Ruminococcaceae_NK4A214_group and Succiniclasticum were highly significantly positively correlated with SGLT1 expression ( $<<0.01)$; Ruminococcaceae_NK4A214_group and Succiniclasticum were significantly positively correlated with $\mathrm{NH}_{3}-\mathrm{N}$ level; and Ruminococcus_ 1 and [Eubacterium]_coprostanoligenes_group were highly significantly negatively correlated with SGLT1 
expression ( $P<0.01)$, but highly significantly positively correlated with ACX and CL activities. This is because the Jun forage quantity and quality are higher than those of the withered grass and ACX activity, the $\mathrm{NH}_{3}-\mathrm{N}$ and total SCFA levels produced by rumen microbial fermentation, and glucose production are higher. The high SGLT1 expression in the rumen epithelium helps to transport D-glucose to the blood faster [40], reduce the glucose concentration in the rumen, and improve or prevent rumen acidosis [41]. It also increases the host energy utilization. Rumen microbes have adapted to highly nutritional forages, so the various cellulose-degrading bacteria in the rumen of Tibetan sheep reach a stable level by Aug, and $\mathrm{CL}$ activity remains at a stable level between Apr and Dec. Therefore, Aug is most conducive to the rumen development and body growth of Tibetan sheep.

The abundance of Rikenellaceae_RC9_gut_group was highest in Dec and lowest in Aug. Rikenellaceae_RC9_gut_group was also a biomarker for Dec samples. Furthermore, random forest analysis showed that Rikenellaceae_RC9_gut_group played important roles in rumen microbial composition. The acetate concentration increased significantly in Oct and Dec, and the propionate and total SCFA concentrations remained at high levels in Oct and Dec. The expression of CLAUDIN4 and ZO1 (which encode rumen epithelial barrier-related proteins that form a physical barrier around the cells to prevent the free passage of small molecules [42]) decreased significantly in Dec, that is, the permeability of the rumen epithelial barrier increased. Succiniclasticum was significantly positively correlated with CLAUDIN4 and ZO1 expression; Rikenellaceae_RC9_gut_group and Fretibacterium were significantly negatively correlated with CLAUDIN4 and ZO1 expression, and significantly positively correlated with acetate concentration.

Further study of the OTU that was found to be unique to the Dec samples (OTU270), as shown in the Venn diagram (Fig. 2A), showed that the species belongs to the phylum Synergistetes, which mainly colonizes anaerobic environments [43] and can ferment amino acids and carry out glycolysis [44,45]. The forage cellulose ratio was slightly higher in Oct than in Aug, and ACX activity was also higher, so the cellulolytic bacteria still produced higher SCFA concentrations (such as a significant increase in acetic acid concentration). Additionally, Synergistetes thoroughly and effectively decomposes forage via amino acid fermentation and glycolysis, thereby providing the host with as much energy as possible. Synergistetes has been reported to be a biomarker of periodontitis in the human oral cavity [46]. Therefore, we speculate that Synergistetes can increase the energy utilization of the host during the period of nutrient deficiency. In this situation, it is beneficial to the host. In Dec, when the quality of forage is further reduced, the host increases the abundance of Synergistetes to provide itself with more energy. At this time, CLAUDIN4 and ZO1 expression also reached their lowest levels, the permeability of the rumen epithelium increased, more small molecules (mainly nutrient molecules) in the rumen easily pass through, and the host obtained more energy. However, when the abundance of Synergistetes increases to a certain level, the rumen epithelium of Tibetan sheep becomes inflamed, the barrier protein structure is gradually destroyed, and the rumen epithelial function decreases. To avoid long-term inflammation, after entering the rejuvenation stage (Apr), the host regulates the microbiota structure by actively regulating rumen epithelial genes, which significantly increases CLAUDIN4 and ZO1 expression, inhibits the passage of 
harmful substances, and returns the CLAUDIN4 and ZO1 expression to normal levels by Aug. This is a typical self-regulatory protection mechanism in Tibetan sheep in alpine regions with insufficient pasture. To prevent the decline in rumen epithelial function of Tibetan sheep observed in Dec, Feb, and Apr due to insufficient forage and the corresponding decrease in production performance, supplementary feeding should be carried out between Dec and Apr of the following year.

In addition, compared to the Dec, Feb, and Apr samples, the Jun samples a significant increase in the number of differential species and the number of biomarkersthe $(P<0.05)$, and a significant change in microbiota structure (the diversity and abundance of rumen microbes). Thus, compared to other months, the rumen microbial composition and structure of the Jun samples underwent substantial adjustments. Based on the KEGG and COG analyses, the METABOLIC pathway also underwent a certain degree of change in Apr to Aug. Therefore, we believe that Jun is the most critical transition stage for Tibetan sheep in the Qinghai-Tibet Plateau, which is of great significance to their growth and development.

\section{Conclusion}

The results showed that there were several differences in rumen fermentation functions of Tibetan sheep in different months. The increases in acetate, propionate, butyrate, and total SCFAs in Oct contributed to rumen development and energy utilization. The increase in $\mathrm{NH}_{3}-\mathrm{N}$ in Jun provided more available nitrogen sources for rumen microorganisms, and the high CL and ACX activities contributed to cellulose degradation. Further, the abundances and diversity of rumen microorganisms changed significantly in different months. Bacteroidetes, Firmicutes, Prevotella_1, Rikenellaceae_RC9_gut_group, and cellulosedecomposing bacteria play important roles in the plateau adaptation of Tibetan sheep, which is mainly related to efficient energy utilization. Additionally, CLAUDIN4 and ZO1 expression in the rumen epithelium were decreased in Dec, which increased the permeability of the rumen epithelial barrier and allowed more small nutrient molecules to pass through. To avoid rumen acidosis, SGLT1 expression in the rumen epithelium was significantly increased in Aug. Correlation analysis showed that rumen fermentation function, microorganisms, and rumen epithelial gene expression were significantly correlated. As forage supply changes throughout the year, the "core microbiota" changes (along with the rumen fermentation mechanisms). Fermentation products and microbes affect the host gene expression, further changing the rumen epithelial permeability and thereby improving energy utilization and rumen homeostasis. It is concluded that the interactions between rumen fermentation products, the microbiota, and host gene expression may play roles in the plateau adaptation mechanisms of Tibetan sheep.

\section{Materials And Methods}

\section{Experimental design and sample collection}

The sample collection site is located in Zuogaimanma Township, Hezuo City, Gannan Tibetan Autonomous Prefecture, Gansu Province, with an altitude of 3300 m. In January 2019, we selected 12 Tibetan sheep ewes from the same herd family that were 1 year old ( \pm 1 month old), in good health, 
similar weight( $35.12 \pm 1.43 \mathrm{~kg})$, traditional natural grazing management, and did not undergo any supplementary feeding. Rumen fluid $(30 \mathrm{~mL})$ was collected by using a sheep gastric tube rumen sampler in February, April, June, August, October, and December, and filtered through 4 layers of sterile gauze. Among them, a part is stored in a $2 \mathrm{~mL}$ sterile cryopreservation tube and stored in liquid nitrogen (for DNA extraction), and the rest is used for the determination of SCFAs, $\mathrm{NH}_{3}-\mathrm{N}$ content, $\mathrm{CL}$ and ACX activities $\left(-20^{\circ} \mathrm{C}\right.$ save). At the same time, Tibetan sheep slaughter experiments were carried out in Apr (return period), Aug (grass period) and Dec (withered grass period), with 3 sheep slaughtered each time. After the neck bloodletting and slaughter, it was dissected immediately, and the rumen tissue was separated. A small piece of rumen abdominal sac tissue was cut out, and the contents on the tissue were quickly rinsed with normal saline. Use blunt scissors to separate the rumen epithelial tissue, place it in a cryotube, store it in liquid nitrogen, and bring it back to the laboratory and store it at $-80{ }^{\circ} \mathrm{C}$ for subsequent RNA extraction. The annual temperature change trend of the sampling site in 2019 is as follows (Fig. 7) :

\section{Determination of rumen fermentation parameters}

SCFAs using a gas chromatograph Shimadzu (GC-2010 plus), using the internal standard method, the internal standard is 2-ethyl butyrate (2EB), Chromatographic column: AT-FFAP $(50 \mathrm{~m} \times 0.32 \mathrm{~mm} \times 0.25 \mu \mathrm{m})$ capillary column. Column heating program: keep the temperature at $60^{\circ} \mathrm{C}$ for $1 \mathrm{~min}$, then increase it to $115^{\circ} \mathrm{C}$ at $5{ }^{\circ} \mathrm{C} / \mathrm{min}$ without retention, and then increase it to $180^{\circ} \mathrm{C}$ at $15^{\circ} \mathrm{C} / \mathrm{min}$, the temperature of the detector is $260{ }^{\circ} \mathrm{C}$, and the temperature of the injection port is $250{ }^{\circ} \mathrm{C}$. Measure the content of $\mathrm{NH}_{3}-\mathrm{N}$ in rumen fluid by spectrophotometer colorimetry; CL activity assay kit and ACX assay kit (Suzhou Keming Biotechnology Co., Ltd., Shanghai, China) were used to determine CL and ACX activities in rumen fluid.

\section{Microbial DNA extraction and 16S rRNA sequencing}

The bacterial DNA extraction kit MN NucleoSpin 96 Soi (Omega, Shanghai, China) was used to extract the rumen fluid microbiome DNA. Use universal primers (338F: 5'-ACTCCTACGGGAGGCAGCA-3' and 806R: 5'GGACTACHVGGGTWTCTAAT-3') to PCR amplification of the V3-V4 region of the 16S rRNA gene hypervariable region [47], The two-step library construction method was used to construct and sequence the rumen microbes [48], and the amplified products were constructed and sequenced on the Illumina MiSeq 2500 (Illumina, San Diego, CA, USA) platform.

\section{Analysis of mRNA levels in rumen epithelial tissue}

Trizol reagent method was used to extract RNA from rumen epithelial tissue of Tibetan sheep (TransGen, Shenyang, China). Use an ultra-micro spectrophotometer (Therm Nano Drop-2000) to determine the concentration and purity of RNA. cDNA synthesis was carried out by reverse transcription kit (HiScript ${ }^{\circledR}$ II Q RT SuperMix for qPCR; Nanjing, China). Use Primer 5.0 software to design primers for SGLT1, CLAUDIN4, and ZO1 genes. The internal reference gene is $\beta$-actin. See Table 3 for primer information. Use Applied Biosystems Q6 real-time fluorescent quantitative PCR instrument for relative quantification of related genes, using a $20 \mathrm{ul}$ reaction system, reaction conditions: $95^{\circ} \mathrm{C}$ pre-denaturation for $30 \mathrm{~s}$; cyclic reaction at $95^{\circ} \mathrm{C}$ for $10 \mathrm{~s}, 60^{\circ} \mathrm{C}$ for $30 \mathrm{~s}, 40$ cycles; dissolution curve conditions $\left(95^{\circ} \mathrm{C}\right.$ for $15 \mathrm{~s}, 60^{\circ} \mathrm{C}$ for $60 \mathrm{~s}$, 
$95^{\circ} \mathrm{C}$ for $\left.15 \mathrm{~s}\right)$. The $\beta$-actin was used as the internal reference gene for calibration, and the data analysis adopted the $2^{-\Delta \Delta C T}$ method.[49]

Table 3

Primer information

\begin{tabular}{|c|c|c|c|c|}
\hline Gene & Primer(5'-3') & Length & $\begin{array}{l}\text { Annealing } \\
\text { temperature }\end{array}$ & Login ID \\
\hline \multirow[t]{2}{*}{$\beta$-actin } & F:AGCCTTCCTTCCTGGGCATGGA & \multirow[t]{2}{*}{$113 b p$} & \multirow[t]{2}{*}{$60^{\circ} \mathrm{C}$} & \multirow[t]{2}{*}{ NM_001009784.3 } \\
\hline & R:GGACAGCACCGTGTTGGCGTAGA & & & \\
\hline \multirow[t]{2}{*}{ SGLT1 } & F:GTGCAGTCAGCACAAAGTGG & \multirow[t]{2}{*}{$198 \mathrm{bp}$} & \multirow[t]{2}{*}{$60^{\circ} \mathrm{C}$} & \multirow[t]{2}{*}{ NM_001009404.1 } \\
\hline & R:CCCGGTTCCATAGGCAAACT & & & \\
\hline \multirow[t]{2}{*}{ CLAUDIN4 } & F:AAGGTGTACGACTCGCTGCT & \multirow[t]{2}{*}{$237 \mathrm{bp}$} & \multirow[t]{2}{*}{$60^{\circ} \mathrm{C}$} & \multirow[t]{2}{*}{ NM_001185017.2 } \\
\hline & R:GACGTTGTTAGCCGTCCAG & & & \\
\hline \multirow[t]{2}{*}{$Z 01$} & F:CGACCAGATCCTCAGGGTAA & \multirow[t]{2}{*}{$161 \mathrm{bp}$} & \multirow[t]{2}{*}{$60^{\circ} \mathrm{C}$} & \multirow[t]{2}{*}{ XM_015101953.2 } \\
\hline & R:AATCACCCACATCGGATTCT & & & \\
\hline
\end{tabular}

The quality of the original sequencing data was evaluated, and the original sequencing reads were denoised and subjected to paired-end read merging (FLASH, version 1.2.11), quality filtering (Trimmomatic, version 0.33), and chimera removal (UCHIME, version 8.1). Usearch software (version 10.0) was employed to cluster the high-quality effective tags, based on $97 \%$ similarity, using the 2013 Greengenes ribosome database (version 13.8). The OTUs were filtered [50], using $0.005 \%$ of all sequences sequenced as the threshold. Using the SILVA database (bacterial 16S), species annotation and taxonomic analysis of OTU were conducted.

Using Mothur (version 1.30), the OTUs were subjected to an alpha diversity analysis (including Shannon index). Rarefaction and species relative abundance accumulation curves were also constructed. Beta diversity analysis was performed using QIIME, with PCoA, non-metric multi-dimensional scaling (NMDS), and ANOSIM being used to compare the species diversity among samples collected in different months. LEfSe analysis was used to identify the biomarkers related to the samples obtained in each month.

Finally, PICRUSt software was used to compare the functional differences related to microbiota species composition (based on $16 \mathrm{~S}$ sequencing data) among samples obtained in different months. First, a differential KEGG pathway analysis was used to identify the differences in the metabolic pathways related to the functional genes of the microbiota in different months. Second, a differential COG function prediction analysis was used to predict the differences in the protein function of the prokaryotes in different months.

\section{Statistical analysis}


SPSS 20.0 (SPSS Inc., Chicago, Illinois, USA) was used for data analysis, and one-way analysis of variance (ANCOVA) was used to analyze rumen fermentation parameters (SCFAs, $\mathrm{NH}_{3}-\mathrm{N}$ content, $\mathrm{CL}$ and ACX activities) and alpha diversity index (Ace Index, Chao1 Index, Shannon Index and Simpson Index) in the rumen of Tibetan sheep at different stages, then Duncan post hoc test. The normality of the data was tested by Shapiro-Wilk test, and the homogeneity of variance was tested by Levene test. LEfSe method was used to evaluate the differences of microbial communities, and the LDA score threshold was 4 . The analysis result data are all expressed as Mean $\pm S D$, and the statistical significance level is $p<0.05$. Spearman was used for correlation test.

\section{Abbreviations}

\begin{tabular}{|ll|}
\hline CL & Cellulase \\
\hline ACX & acidic xylanase \\
\hline KEGG & Kyoto Encyclopedia of Genes and Genomes \\
\hline COG & Clusters of Orthologous Groups of proteins \\
\hline PCoA & principal coordinates analysis \\
\hline NMDS & non-metric multi-dimensional scaling \\
\hline ANOSIM & analysis of similarities \\
\hline LDA & Linear discriminant analysis \\
\hline LEfSe & LDA effect size \\
\hline ANCOVA & one-way analysis of variance \\
\hline
\end{tabular}

\section{Declarations}

\section{Acknowledgements}

We thank Zhidong Zhao and Bingang Shi for helpful discussions and comments. We also thank Zengyuan Qi and Jianqiang Zhou for help with research support.

\section{Authors' contributions}

Xiu Liu and Yuzhu Luo designed the study; Weibing Lv, Hao Shi, Hong Wei, Xinyu Guo and Xiaoning Pu performed all experiments; Jiqing Wang, Jiang Hu, Shaobin Li and Yuzhu Sha analysed the data; Weibing Lv drafted the paper with contributions from Xiu Liu and Yuzhu Sha.

\section{Funding}

We thank for the financial support: Supporting funds for youth mentor of Gansu Agricultural University (GAU-QDFC-2018-11) and Innovation Funds of Gansu province institution of higher learning (2020A-52). 
Availability of data and materials

Raw sequence data associated with is being uploaded to NCBI.

\section{Ethics approval and consent to participate}

All studies involving animal were carried out in accordance with the regulations for the Administration of Affairs Concerning Experimental Animal (Ministry of Science and Technology, China; revise in June 2004), and sample collection protocols were approved by the Livestock Care Committee of Gansu Agricultural University (Approval No. GAU-LC-2020-27).

\section{Consent for publication}

Not applicable.

\section{Competing interests}

The authors have no conflicts of interest.

\section{Author details}

College of Animal Science and Technology, Gansu Agricultural University, Lanzhou, 730070, China

\section{References}

1. Guo P, Gao P, Li F, Chang S, Wang Z, Yan T, et al. Prediction of Metabolizable Energy Concentrations of Herbage in the Qinghai-Tibetan Plateau Using Tibetan Sheep Digestibility Data. Animals : an open access journal from MDPI, 2020; 10(3): 376.

2. Liu H, Hu L, Han X, Zhao N, Xu T, Ma L, et al. Tibetan Sheep Adapt to Plant Phenology in Alpine Meadows by Changing Rumen Microbial Community Structure and Function. Frontiers in microbiology, 2020; 11: 587558.

3. Amato K R, Leigh S R, Kent A, Mackie R I, Yeoman C J, Stumpf R M, et al. The gut microbiota appears to compensate for seasonal diet variation in the wild black howler monkey (Alouatta pigra). Microbial ecology, 2015; 69(2): 434-443.

4. Sun B, Wang X, Bernstein S, Huffman M A, Xia D P, Gu Z, et al. Marked variation between winter and spring gut microbiota in free-ranging Tibetan Macaques (Macaca thibetana). Scientific reports, 2016; 6: 26035.

5. Foster K R, Schluter J, Coyte K Z, Rakoff-Nahoum S. The evolution of the host microbiome as an ecosystem on a leash. Nature, 2017;. 548(7665): 43-51.

6. Michaudel C, Sokol H. The Gut Microbiota at the Service of Immunometabolism. Cell metabolism, 2020; 32(4): 514-523. 
7. Pigneur B, Sokol H. Fecal microbiota transplantation in inflammatory bowel disease: the quest for the holy grail. Mucosal immunology, 2016; 9(6): 1360-1365.

8. Lin X, Wang J, Hou Q, Wang Y, Hu Z, Shi K, et al. Effect of hay supplementation timing on rumen microbiota in suckling calves. MicrobiologyOpen, 2018; 7(1): e00430.

9. E Edwards J, R McEwan N, J Travis A, John Wallace R. 16S rDNA library-based analysis of ruminal bacterial diversity. Antonie van Leeuwenhoek, 2004; 86(3): 263-281.

10. Lin L, Xie F, Sun D, Liu J, Zhu W, Mao S. Ruminal microbiome-host crosstalk stimulates the development of the ruminal epithelium in a lamb model. Microbiome, 2019; 7(1): 83.

11. Lin L, Xie F, Sun D, Liu J, Zhu W, Mao S. Ruminal microbiome-host crosstalk stimulates the development of the ruminal epithelium in a lamb model. Microbiome, 2019; 7(1): 83.

12. Hooper L V, Midtvedt T, Gordon J I. How host-microbial interactions shape the nutrient environment of the mammalian intestine. Annual review of nutrition, 2002; 22: 283-307.

13. Malmuthuge N, Guan L L. Understanding host-microbial interactions in rumen: searching the best opportunity for microbiota manipulation. Journal of animal science and biotechnology, 2017; 8: 8.

14. Allaire J M, Crowley S M, Law H T, Chang S Y, Ko H J, Vallance B A. The Intestinal Epithelium: Central Coordinator of Mucosal Immunity. Trends in immunology, 2018; 39(9): 677-696.

15. Martin-Gallausiaux C, Larraufie P, Jarry A, Béguet-Crespel F, Marinelli L, Ledue F, Lapaque N. Butyrate Produced by Commensal Bacteria Down-Regulates Indolamine 2,3-Dioxygenase 1 (ID0-1) Expression via a Dual Mechanism in Human Intestinal Epithelial Cells. Frontiers in immunology, 2018; 9: 2838.

16. Zhang K, Tu Y, Gao L, Meng M, Bai Y. Replacement of grains with soybean hulls ameliorates SARAinduced impairment of the colonic epithelium barrier function of goats. BMC veterinary research, 2018; 14(1): 376.

17. Furuse M, Fujita K, Hiiragi T, Fujimoto K, Tsukita S. Claudin-1 and - 2: novel integral membrane proteins localizing at tight junctions with no sequence similarity to occludin. The Journal of cell biology, 1998; 141(7): 1539-1550.

18. Stevenson B R, Siliciano J D, Mooseker M S, Goodenough D A. Identification of ZO-1: a high molecular weight polypeptide associated with the tight junction (zonula occludens) in a variety of epithelia. The Journal of cell biology, 1986; 103(3): 755-766.

19. Aschenbach JR, Bhatia SK, Pfannkuche H, Gäbel G. Glucose is absorbed in a sodium-dependent manner from forestomach contents of sheep. J Nutr. 2000; 130(11):2797 - 801.

20. Russell J B, Rychlik J L. Factors that alter rumen microbial ecology. Science (New York, N. Y.), 292(5519), 2001; 1119-1122.

21. Long R J, Apori S O, Castro F B, Rskov E R. Feed value of native forages of the tibetan plateau of china. Animal Feed Science and Technology, 1999; 80(2): 101-113.

22. Liu H, Hu L, Han X, Zhao N, Xu T, Ma L, Xu S. Tibetan Sheep Adapt to Plant Phenology in Alpine Meadows by Changing Rumen Microbial Community Structure and Function. Frontiers in microbiology, 2020; 11: 587558. 
23. Liu H, Xu T, Xu S, Ma L, Han X, Wang X, Zhao X. Effect of dietary concentrate to forage ratio on growth performance, rumen fermentation and bacterial diversity of Tibetan sheep under barn feeding on the Qinghai-Tibetan plateau. PeerJ, 2019; 7: e7462.

24. Qin W, Song P, Lin G., Huang Y, Wang L, Zhou X, Zhang T. Gut Microbiota Plasticity Influences the Adaptability of Wild and Domestic Animals in Co-inhabited Areas. Frontiers in microbiology, 2020; 11: 125.

25. Tajima K, Aminov R I, Nagamine T, Matsui H, Nakamura M, Benno Y. Diet-dependent shifts in the bacterial population of the rumen revealed with real-time PCR. Applied and environmental microbiology, 2001; 67(6): 2766-2774.

26. Kim M, Morrison M, Yu Z. Status of the phylogenetic diversity census of ruminal microbiomes. FEMS microbiology ecology, 2011; 76(1): 49-63.

27. Cunha I S, Barreto C C, Costa O Y, Bomfim M A, Castro A P, Kruger R H, Quirino B F. Bacteria and Archaea community structure in the rumen microbiome of goats (Capra hircus) from the semiarid region of Brazil. Anaerobe, 2011; 17(3): 118-124.

28. Jami E, White B A, Mizrahi I. Potential role of the bovine rumen microbiome in modulating milk composition and feed efficiency. PloS one, 2014; 9(1): e85423.

29. Stevenson D M, Weimer P J. Dominance of Prevotella and low abundance of classical ruminal bacterial species in the bovine rumen revealed by relative quantification real-time PCR. Applied microbiology and biotechnology, 2007; 75(1): 165-174.

30. Seshadri R, Leahy S C, Attwood G T, Teh K H, Lambie S C, Cookson A L, Kelly W J. Cultivation and sequencing of rumen microbiome members from the Hungate1000 Collection. Nature biotechnology, 2018; 36(4): 359-367.

31. Peng B, Huang S, Liu T, Geng A. Bacterial xylose isomerases from the mammal gut Bacteroidetes cluster function in Saccharomyces cerevisiae for effective xylose fermentation. Microbial cell factories, 2015; 14: 70.

32. Yang L Y, Chen J, Cheng X L, Xi D M, Yang S L, Deng W D, Mao H M. Phylogenetic analysis of $16 S$ rRNA gene sequences reveals rumen bacterial diversity in Yaks (Bos grunniens). Molecular biology reports, 2015; 37(1): 553-562.

33. Abdul Rahman N, Parks D H, Vanwonterghem I, Morrison M, Tyson G W, Hugenholtz P. A Phylogenomic Analysis of the Bacterial Phylum Fibrobacteres. Frontiers in microbiology, 2016; 6: 1469.

34. Ley R E, Peterson D A, Gordon J I. Ecological and evolutionary forces shaping microbial diversity in the human intestine. Cell, 2006; 124(4): 837-848.

35. Pitta D W, Pinchak W E, Indugu N, Vecchiarelli B, Sinha R, Fulford J D. Metagenomic Analysis of the Rumen Microbiome of Steers with Wheat-Induced Frothy Bloat. Frontiers in microbiology, 2016; 7: 689.

36. Henderson G, Cox F, Ganesh S, Jonker A, Young W, Global Rumen Census Collaborators, Janssen P $\mathrm{H}$. Rumen microbial community composition varies with diet and host, but a core microbiome is 
found across a wide geographical range. Scientific reports, 2015; 5: 14567.

37. Shi W, Moon C D, Leahy S C, Kang D, Froula J, Kittelmann S, Rubin E M. Methane yield phenotypes linked to differential gene expression in the sheep rumen microbiome. Genome research, 2014; 24(9): 1517-1525.

38. Bergman $E N$. Energy contributions of volatile fatty acids from the gastrointestinal tract in various species. Physiological reviews, 1990; 70(2): 567-590.

39. Hamer H M, Jonkers D, Venema K, Vanhoutvin S, Troost F J, Brummer R J. Review article: the role of butyrate on colonic function. Alimentary pharmacology \& therapeutics, 2008; 27(2): 104-119.

40. Aschenbach J R, Wehning H, Kurze M, Schaberg E, Nieper H, Burckhardt G, Gäbel G. Functional and molecular biological evidence of SGLT-1 in the ruminal epithelium of sheep. American journal of physiology. Gastrointestinal and liver physiology, 2000; 279(1): G20-G27.

41. Aschenbach J R, Borau T, Gäbel G. Glucose uptake via SGLT-1 is stimulated by beta(2)-adrenoceptors in the ruminal epithelium of sheep. The Journal of nutrition, 2002; 132(6): 1254-1257.

42. Anderson $\mathrm{J} \mathrm{M}$, Van Itallie $\mathrm{C} M$. Tight junctions and the molecular basis for regulation of paracellular permeability. The American journal of physiology, 1995; 269(4 Pt 1): G467-G475.

43. Bhandari V, Gupta R S. Molecular signatures for the phylum Synergistetes and some of its subclades. Antonie van Leeuwenhoek, 2012; 102(4): 517-540.

44. Downes J, Vartoukian S R, Dewhirst F E, Izard J, Chen T, Yu W H, Wade W G. Pyramidobacter piscolens gen. nov., sp. nov., a member of the phylum 'Synergistetes' isolated from the human oral cavity. International journal of systematic and evolutionary microbiology, 2009; 59(Pt 5): 972-980.

45. Jumas-Bilak E, Roudière L, Marchandin H. Description of 'Synergistetes' phyl. nov. and emended description of the phylum 'Deferribacteres' and of the family Syntrophomonadaceae, phylum 'Firmicutes'. International journal of systematic and evolutionary microbiology, 2009; 59(Pt 5): 10281035.

46. Khemwong T, Kobayashi H, Ikeda Y, Matsuura T, Sudo T, Kano C, Izumi Y. Fretibacterium sp. human oral taxon 360 is a novel biomarker for periodontitis screening in the Japanese population. PloS one, 2019; 14(6): e0218266.

47. Dunphy-Doherty F, O'Mahony S M, Peterson V L, O'Sullivan O, Crispie F, Cotter P D, Fone K. Postweaning social isolation of rats leads to long-term disruption of the gut microbiota-immune-brain axis. Brain, behavior, and immunity, 2018; 68: 261-273.

48. Miya M, Sato Y, Fukunaga T, Sado T, Poulsen J Y, Sato K, Iwasaki, W MiFish. A set of universal PCR primers for metabarcoding environmental DNA from fishes: detection of more than 230 subtropical marine species. Royal Society open science, 2015; 2(7): 150088.

49. Livak K J, Schmittgen T D. Analysis of Relative Gene Expression Data Using Real-Time Quantitative PCR and the $2^{-\triangle \triangle C T}$ Method. Methods, 2001; 25, 402-408.

50. Bokulich N A, Subramanian S, Faith J J, Gevers D, Gordon J I, Knight R, Caporaso J G. Quality-filtering vastly improves diversity estimates from Illumina amplicon sequencing. Nature methods, 2013; 
10(1): 57-59.

Figures

A

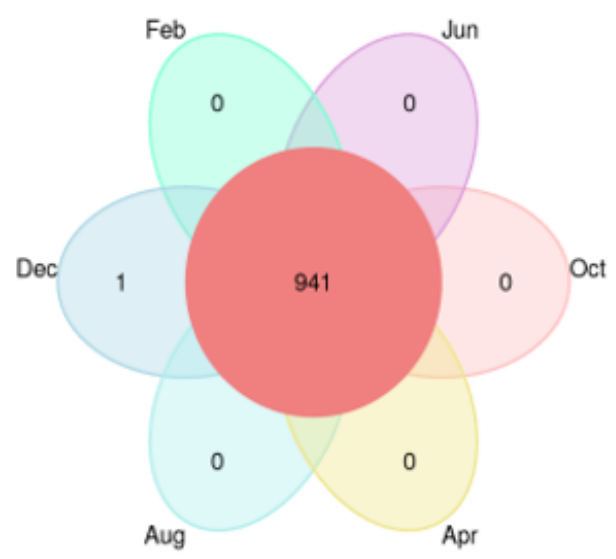

C

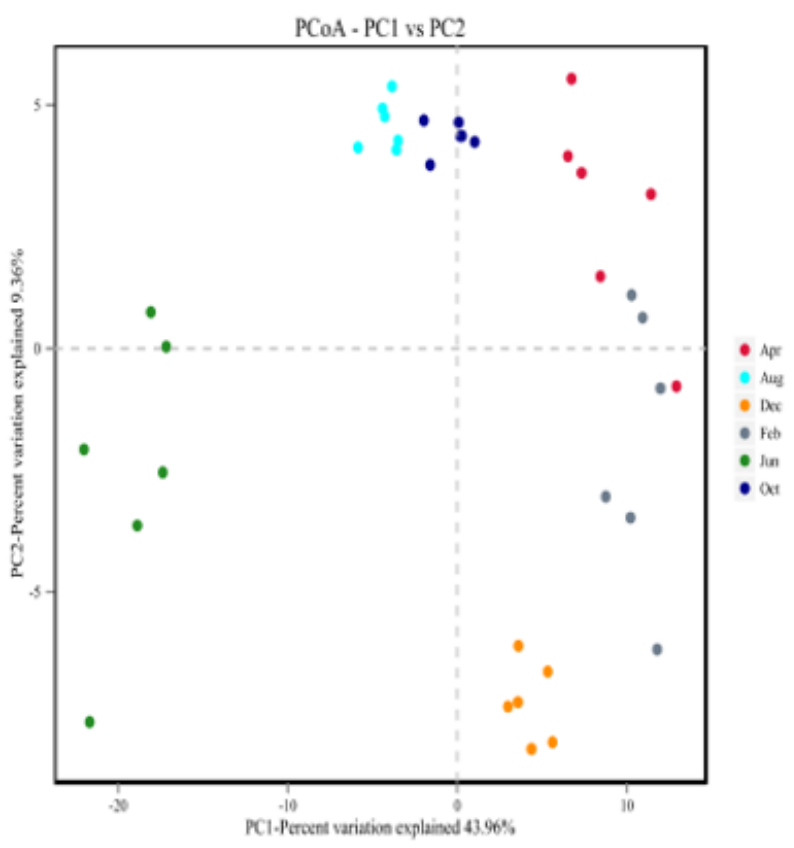

\section{B}

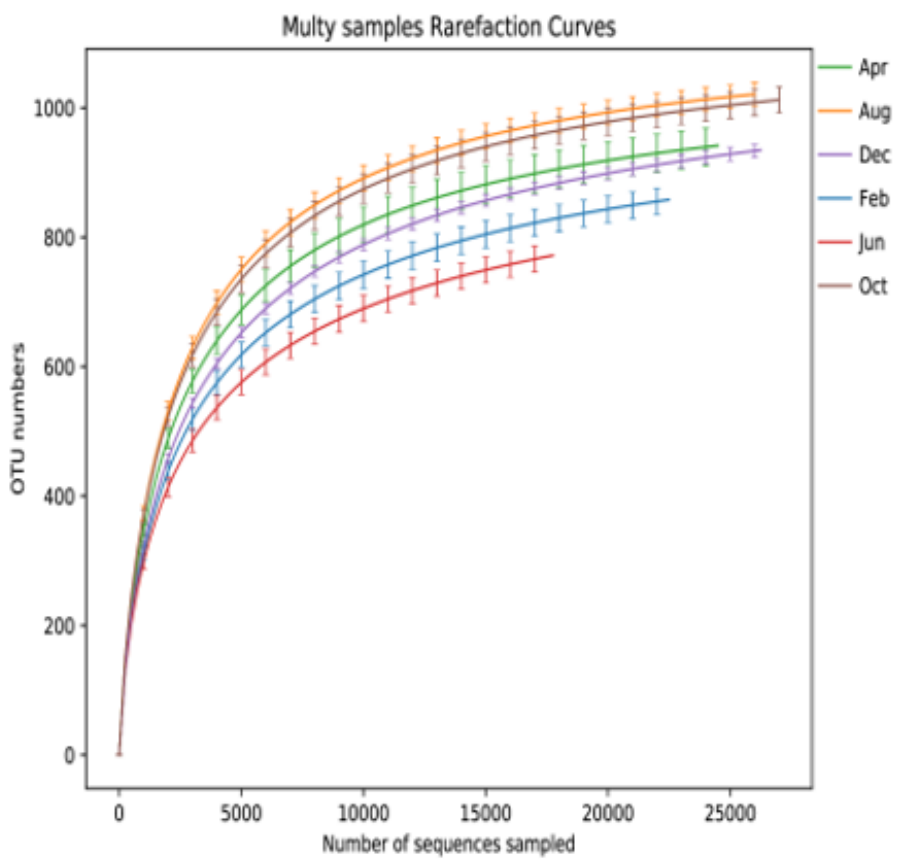

D

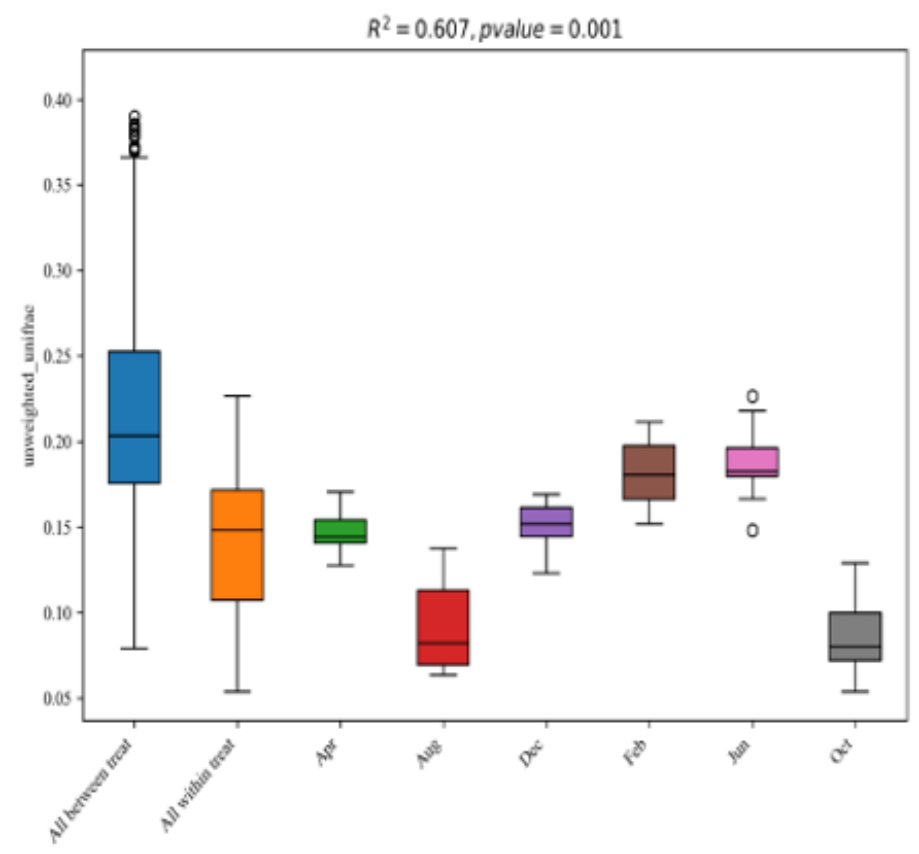

Figure 1

A Distribution map of microbial OTU B Dilution curve C PCoA analysis D Anosim analysis box plot 
A

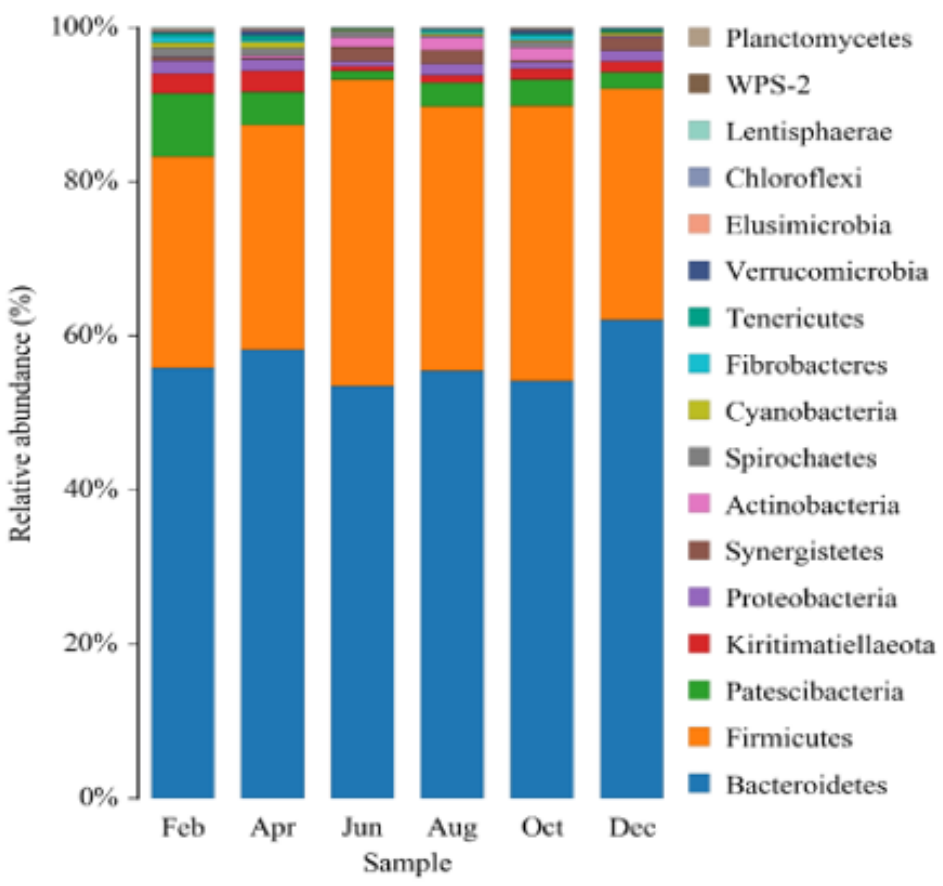

C

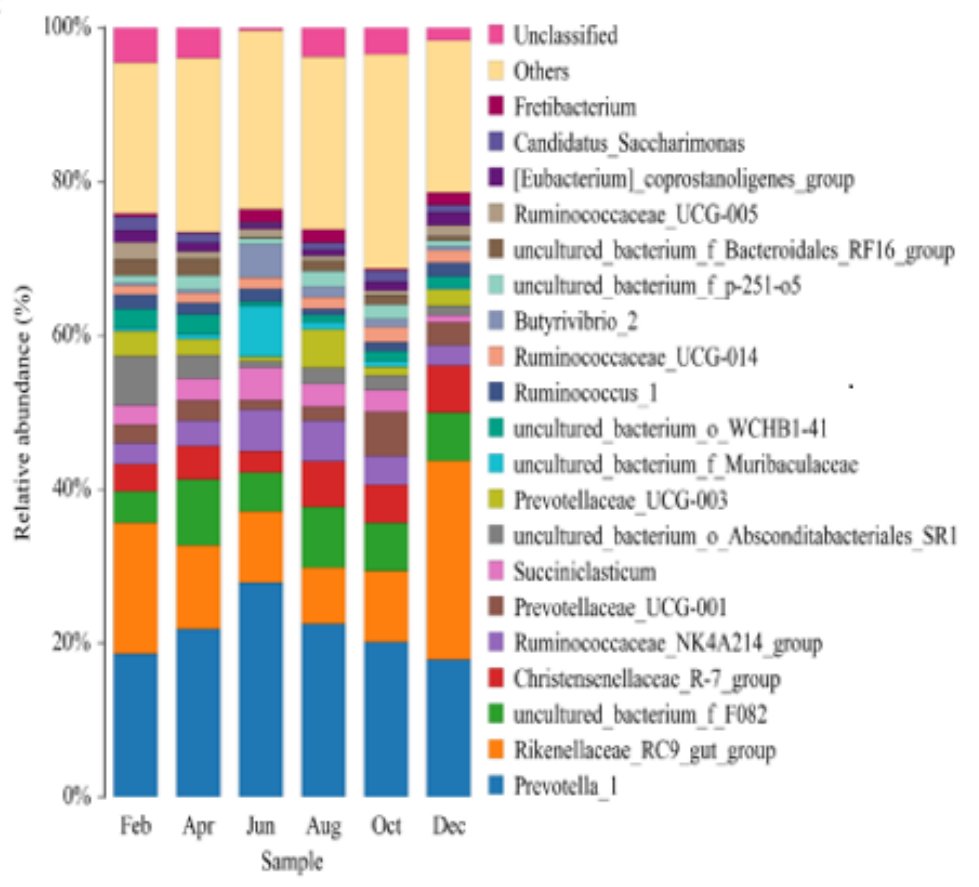

B

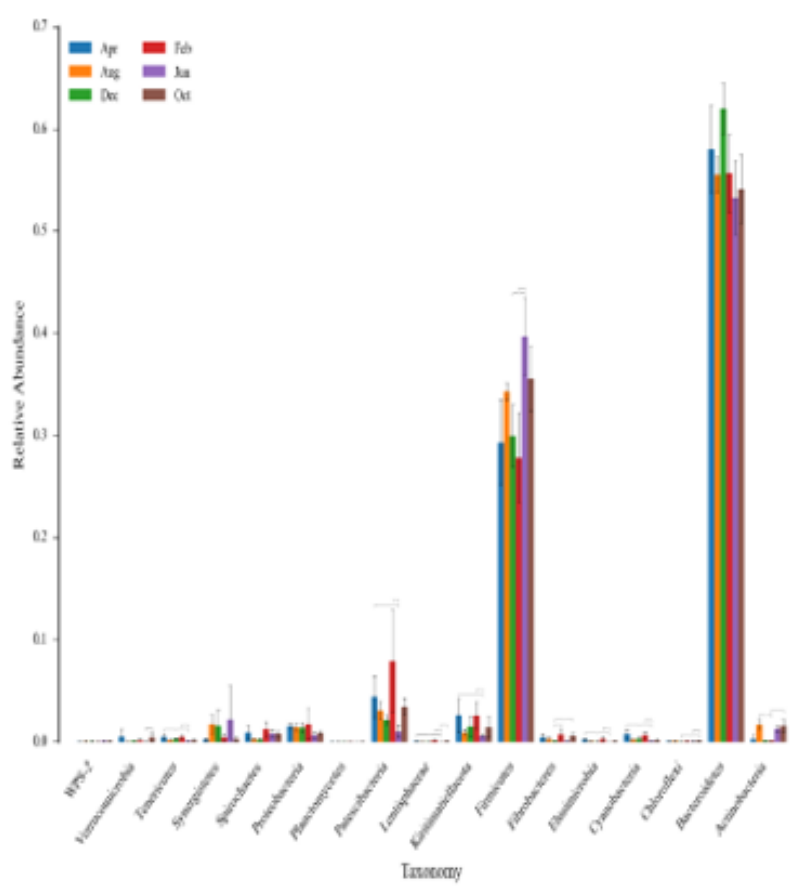

D

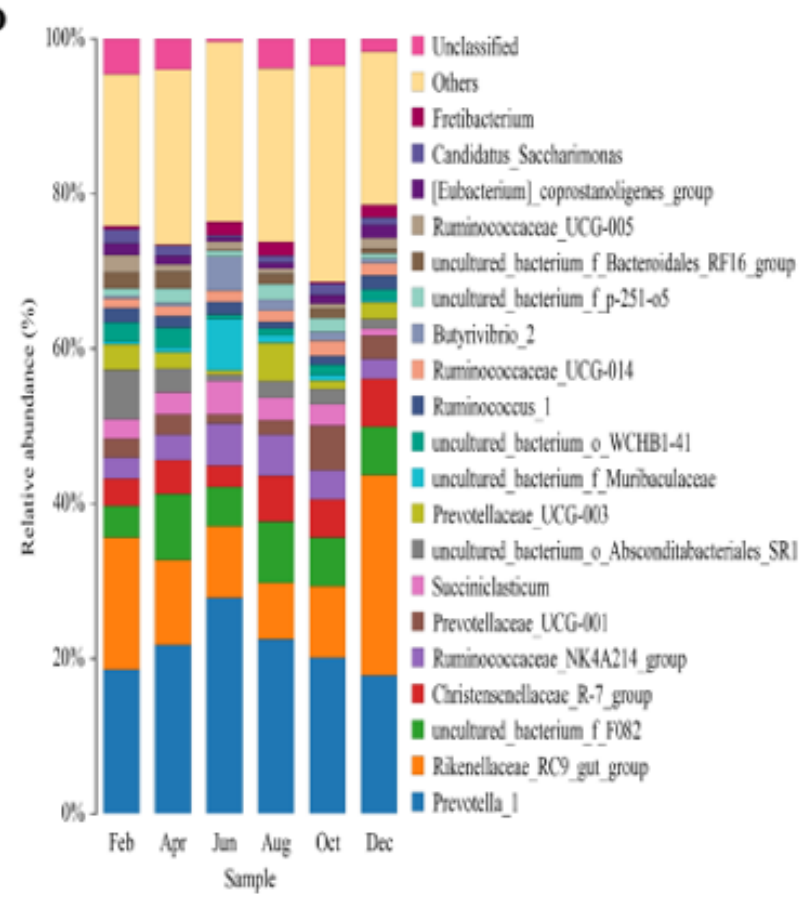

Figure 2

A Phylum species composition histogram B Phylum rank sum test analysis histogram C Genus species composition histogram D Genus rank sum test analysis histogram 


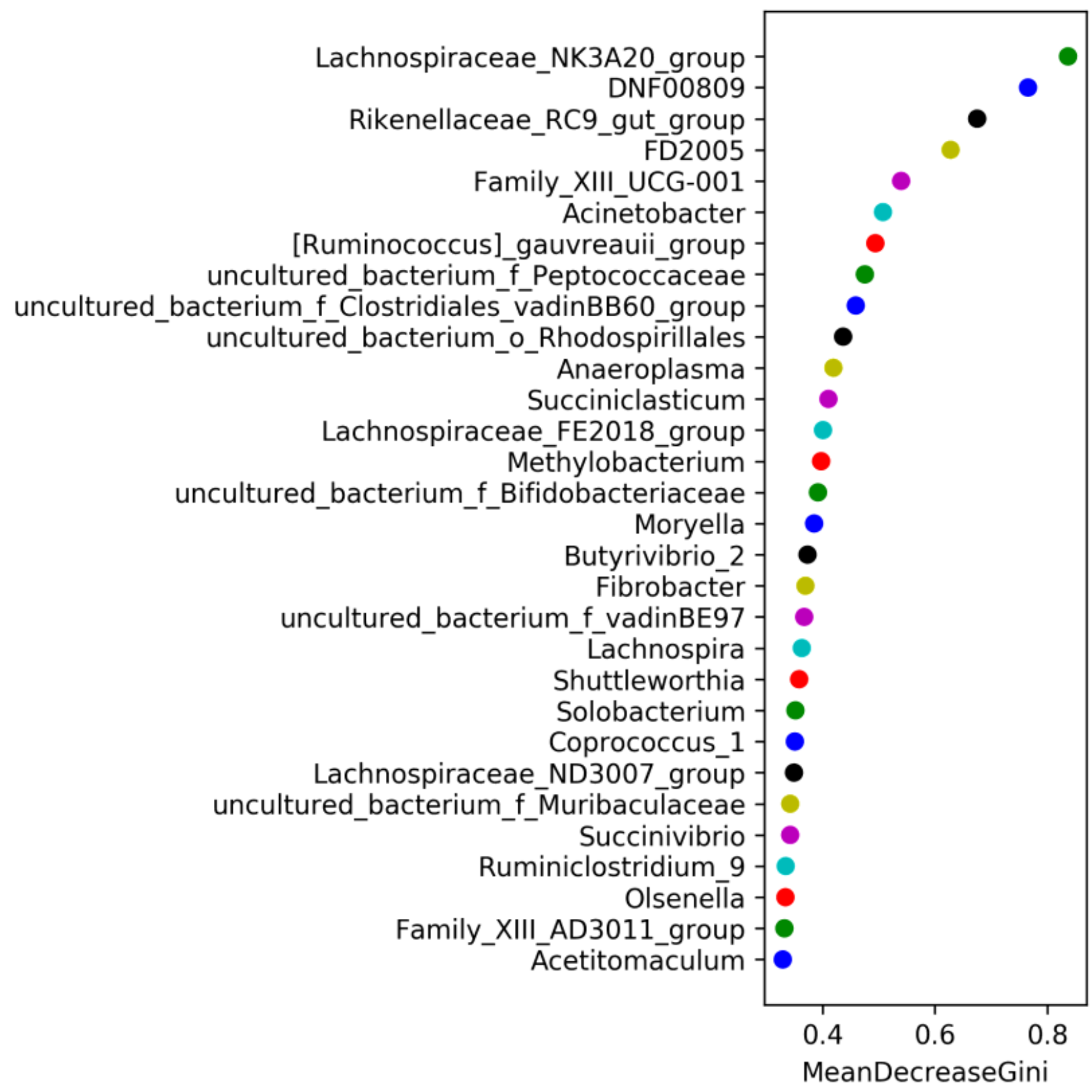

Figure 3

Random forest analysis of microorganisms at the genus level in different periods 

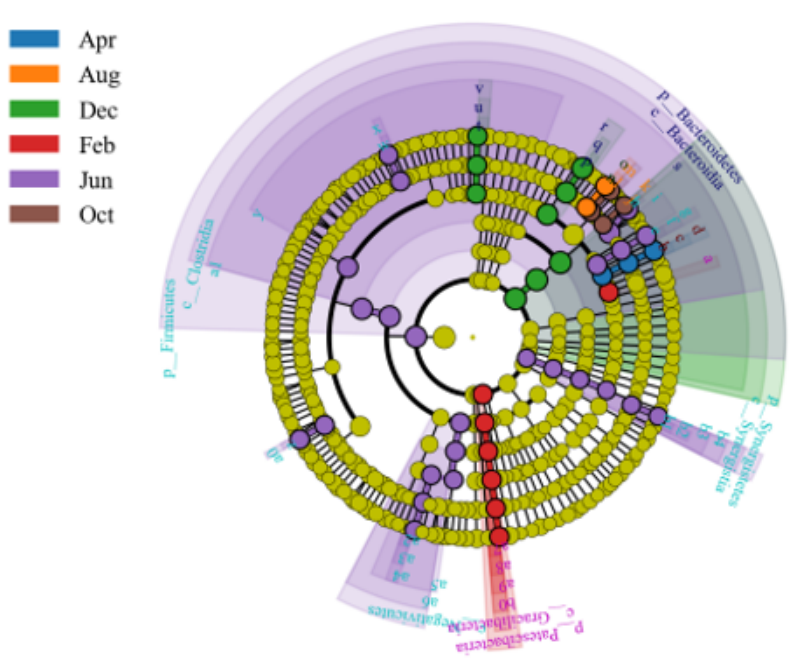

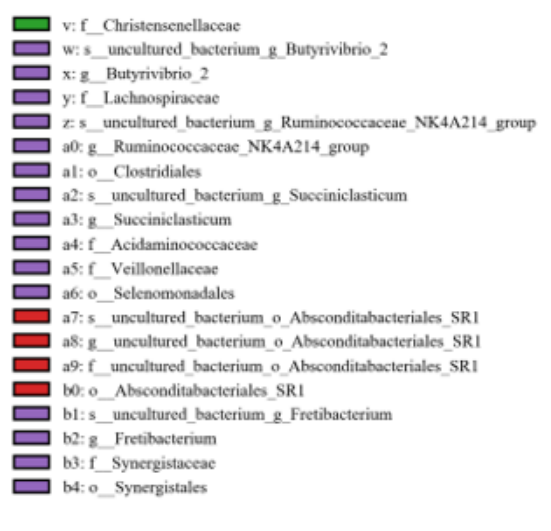

Figure 4

LEfSe analysis evolutionary branch diagram

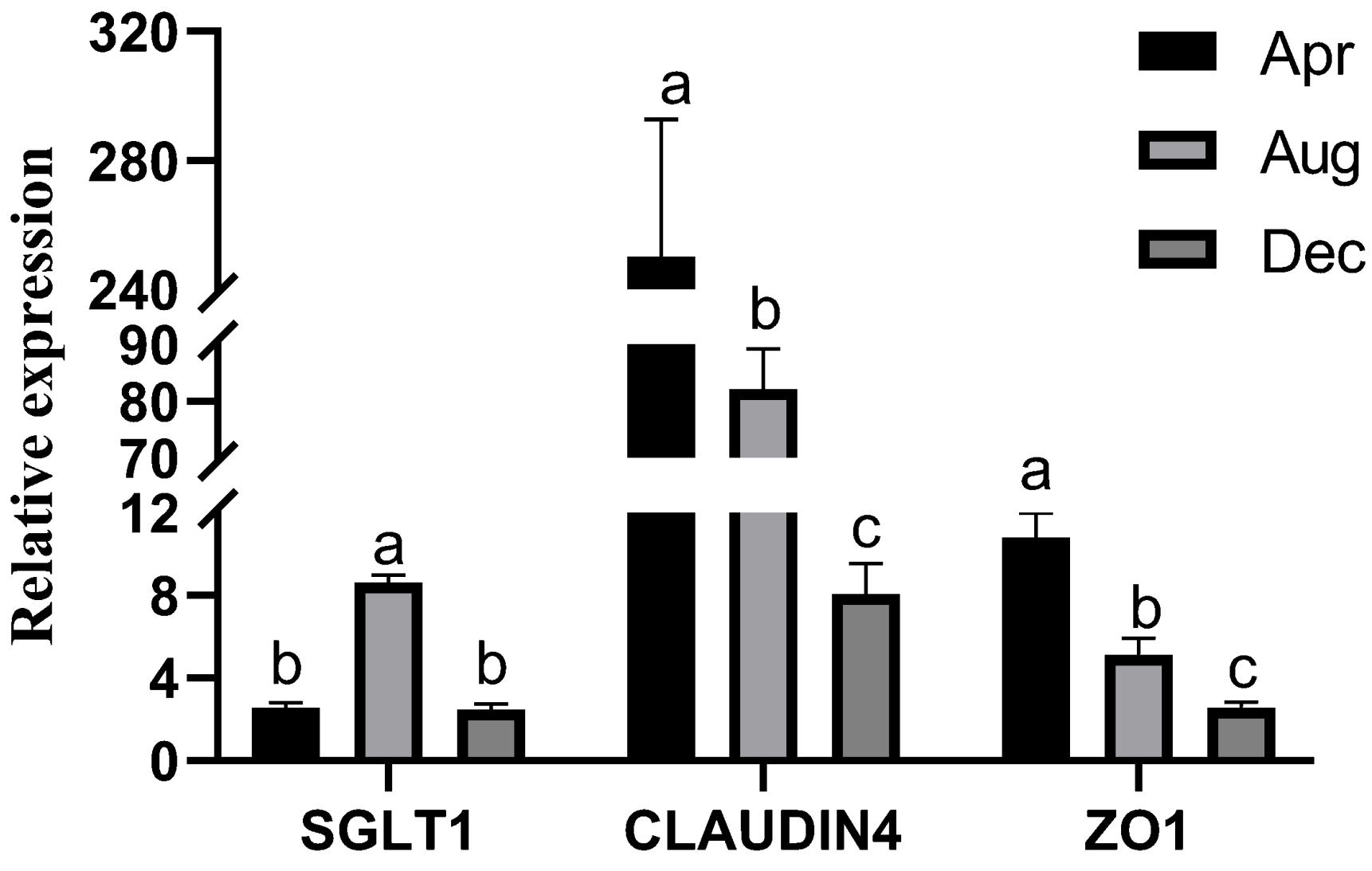

Figure 5

Expression of SGLT1, CLAUDIN4 and Z01 genes in rumen epithelial tissue 


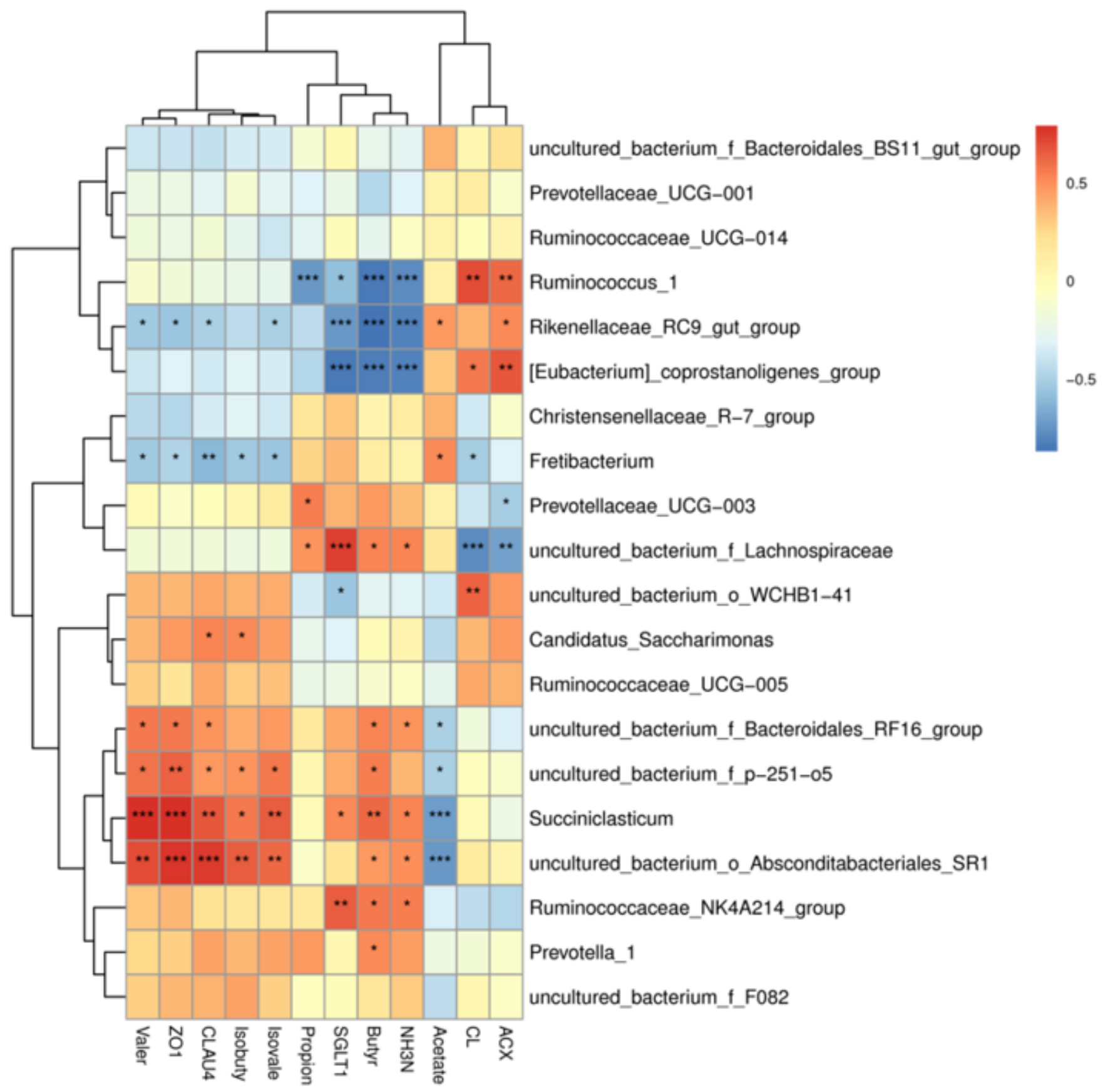

Figure 6

Rumen fermentation-microbe-host gene expression correlation heat map 


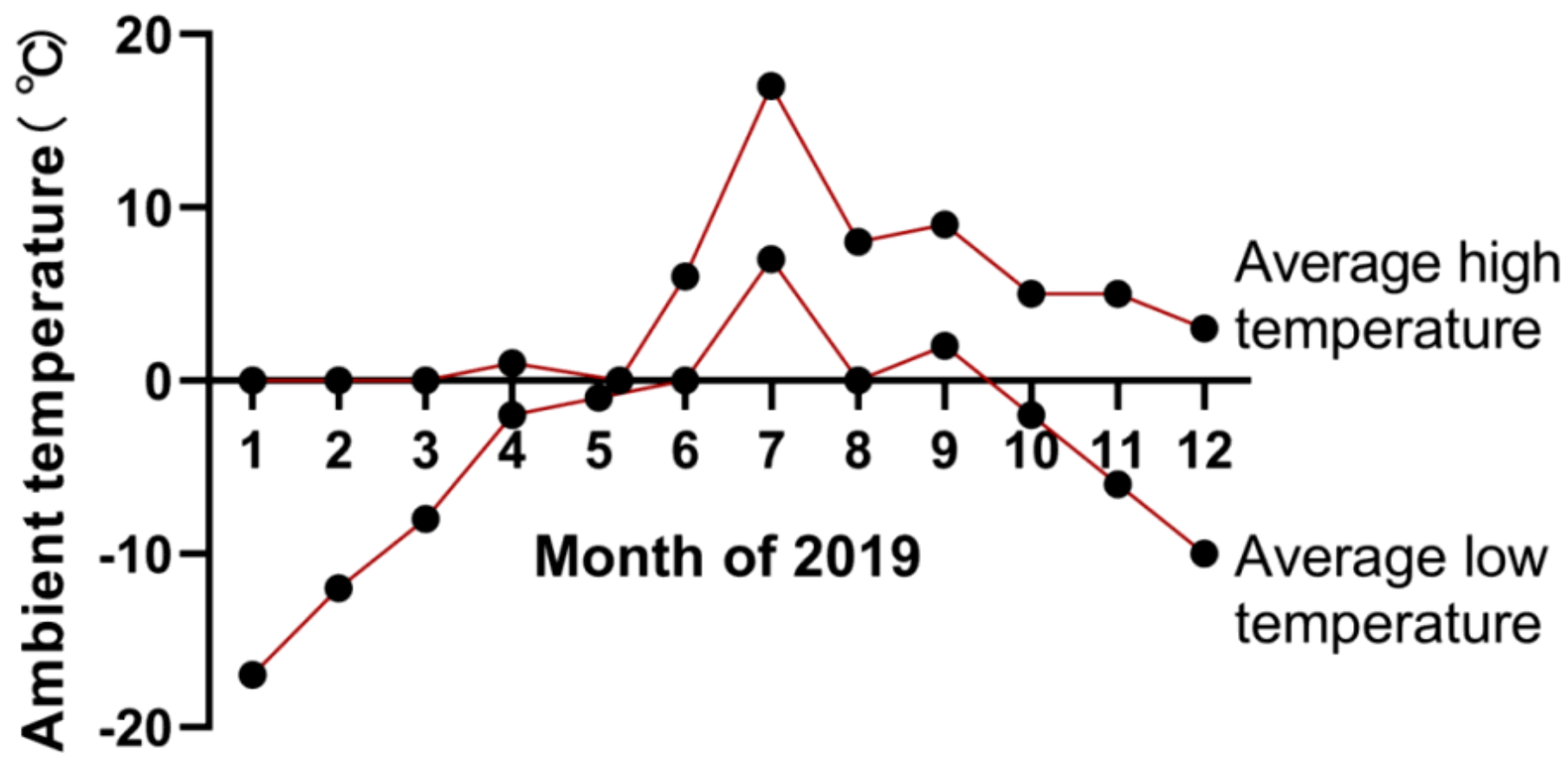

Figure 7

Ambient temperature trend map of the sampling site in 2019 